\title{
Cat-to-Human Transmission of Mycobacterium bovis, United Kingdom
}

\author{
Catherine M. O'Connor, Muhammad Abid, ${ }^{1}$ \\ Amanda L. Walsh, Behrooz Behbod, ${ }^{2}$ \\ Tony Roberts, Linda V. Booth, H. Lucy Thomas, \\ Noel H. Smith, Eleftheria Palkopoulou, \\ James Dale, Javier Nunez-Garcia, ${ }^{3}$ Dilys Morgan
}

Human infection with Mycobacterium bovis is reported infrequently in the United Kingdom. Most cases involve previous consumption of unpasteurized milk. We report a rare occurrence of 2 incidents of cat-to-human transmission of $M$. bovis during a cluster of infection in cats.

$\mathrm{I}^{\mathrm{n}}$ n the United Kingdom, Mycobacterium bovis infection in humans is relatively rare (1), and most cases involve previous exposure to well-recognized risk factors, such as unpasteurized milk (2). However, with $>4,500$ new cases reported in cattle herds each year during 2014-2018 (3), $M$. bovis remains a major issue for animal health in large parts of England and Wales.

Cats are considered spillover hosts of $M$. bovis in the United Kingdom. During 2002-2010, <30 feline cases of M. bovis infection were confirmed by laboratory culture each year by the Animal and Plant Health Agency M. bovis Reference Laboratory (4). Cases of M. bovis infection in cats are generally restricted to areas to which bovine tuberculosis (TB) is endemic (5), where infected cattle and wildlife have the potential to introduce $M$. bovis into cat populations. Most reported feline cases of M. bovis infection in the United Kingdom are sporadic, and outside the occasional household-linked cases, spatially or temporally linked clusters are unusual.

The potential for cat-to-human transmission of M. bovis has always been recognized. Although concurrent infection in cats and humans in the same household has been reported (6), and reports of potential transmission exist (7), documented transmission events have not been clearly

Author affiliations: Public Health England, London, UK

(C.M. O'Connor, A.L. Walsh, H.L. Thomas, D. Morgan); Public Health England, Chilton, UK (M. Abid, B. Behbod); Animal and Plant Health Agency, Oxford, UK (T. Roberts); Public Health England, Fareham, UK (L.V. Booth); Animal and Plant Health Agency, Weybridge, UK (N.H. Smith, E. Palkopoulou, J. Dale, J. Nunez-Garcia)

DOI: https://doi.org/10.3201/eid2512.190012 described. We report a rare occurrence of microbiologically and genetically confirmed cat-to-human transmission of M. bovis.

\section{The Study}

During December 2012-March 2013, a veterinary practice in Berkshire, England, diagnosed 7 confirmed (culture from lesions or wounds) and 2 suspected (clinically compatible) cases of $M$. bovis disease in domestic cats. One additional suspected case was identified after an interview with an affected household. No samples were available from any of the suspected cases for confirmation for this study. The 10 cats belonged to 9 separate households, of which 6 were $\leq 250 \mathrm{~m}$ from each other. All cats had severe systemic infection, including discharging lymph nodes, nonhealing or discharging infected wounds, and radiographic pulmonary signs. Isolates from the culture-confirmed cases were of the same genotype $(10: \mathrm{u})$, were similar by whole-genome sequencing, and separated into 2 clusters by a single informative polymorphism (8). Veterinary investigations did not determine the source of infection, but the source was believed to be infected wildlife, most likely rodents or badgers, for at least some of the cats. Further information on the investigation into this cluster of infection in cats has been reported ( 8 ).

The unusual size and severity of the cluster of feline M. bovis cases led to the decision that TB screening (9) would be offered to all human household members and others who had close contact with the infected cats. Local Health Protection Teams of Public Health England identified 39 human contacts; 24 accepted TB screening. Three persons (person A, 13 years of age; person B, 18 years of age; and person $\mathrm{C}, 39$ years of age) were positive for latent TB infection (LTBI) by a combination of interferon- $\gamma$ release assays and Mantoux screening tests; none showed evidence of active disease.

These 3 persons with LTBI reported close contact with 2 cats with culture-confirmed $M$. bovis infection while the

${ }^{1}$ Current affiliation: United Arab Emirates University, Al Ain, United Arab Emirates.

${ }^{2}$ Current affiliations: Communicable Disease Surveillance Centre, Public Health Wales, Cardiff, Wales, UK, and Cardiff University, Cardiff.

${ }^{3}$ Current affiliation: Genomics Medicine Ireland, Dublin, Ireland. 
cats had clinical disease (1 had a discharging nonhealing wound and 1 had a discharging lymph node). Apart from contact with the infected cats, no other risk factors for $M$. bovis or $M$. tuberculosis exposure were identified. Because it was not possible to isolate the causative organism from cats or persons with LTBI or identify the likely exposure or source of infection, it was not possible to determine whether transmission of M. bovis from these affected cats to their human contacts had occurred. All 3 persons with LTBI were offered chemoprophylaxis, but only 1 (person A) accepted. All 3 persons were advised to go to local health services if any symptoms potentially indicative of active TB developed.

Six months after initial screening, person B was medically assessed because of nonspecific abdominal pain. Chest radiographs showed evidence of pathologic changes potentially indicative of TB. M. bovis was isolated from pleural biopsy samples. Shortly after person B had clinical illness, a nonhousehold human contact (person D, 20 years of age) of person B and their cat also had $M$. bovis isolated from pleural biopsy samples after reporting chest pain and fever. Person D had initially declined screening. Persons B and D had close contact with the infected cat while it was systemically ill (including a discharging wound). The cat died before $M$. bovis infection was diagnosed. Both persons completed a 9-month course of rifampin, isoniazid, pyrazinamide, and ethambutol and responded well to treatment (10).

Molecular analysis showed that persons B and D, who had active $M$. bovis disease, and the cat all had M. bovis isolates of the same genotype. Whole-genome sequencing of samples from one of the humans and the cat showed that their isolates were indistinguishable (Figure). (Sequencing was not possible for the isolate from the second human patient.) This evidence, coupled with the timeline of onset of disease in the cat (March 2013) and its human contacts (October 2013), and the lack of any other risk factors for exposure to $M$. bovis, indicated that the cat was the likely source of infection for these 2 affected persons.

\section{Conclusions}

Before this incident, the absence of confirmed reports of human cases of $M$. bovis infection acquired from pet cats led us to believe that the risk for cat-to-human transmission was negligible. Thus, no public health action was warranted. However, with the evidence of transmission from 1 cat to these 2 patients, the risk for spread of $M$. bovis from cats to their human contacts was increased from negligible to low (11). Cats with clinical signs compatible with disseminated disease are believed to have the greatest risk to humans, most likely by ingestion from a contaminated environment, following handling of discharges from exudative tuberculous lesions, or

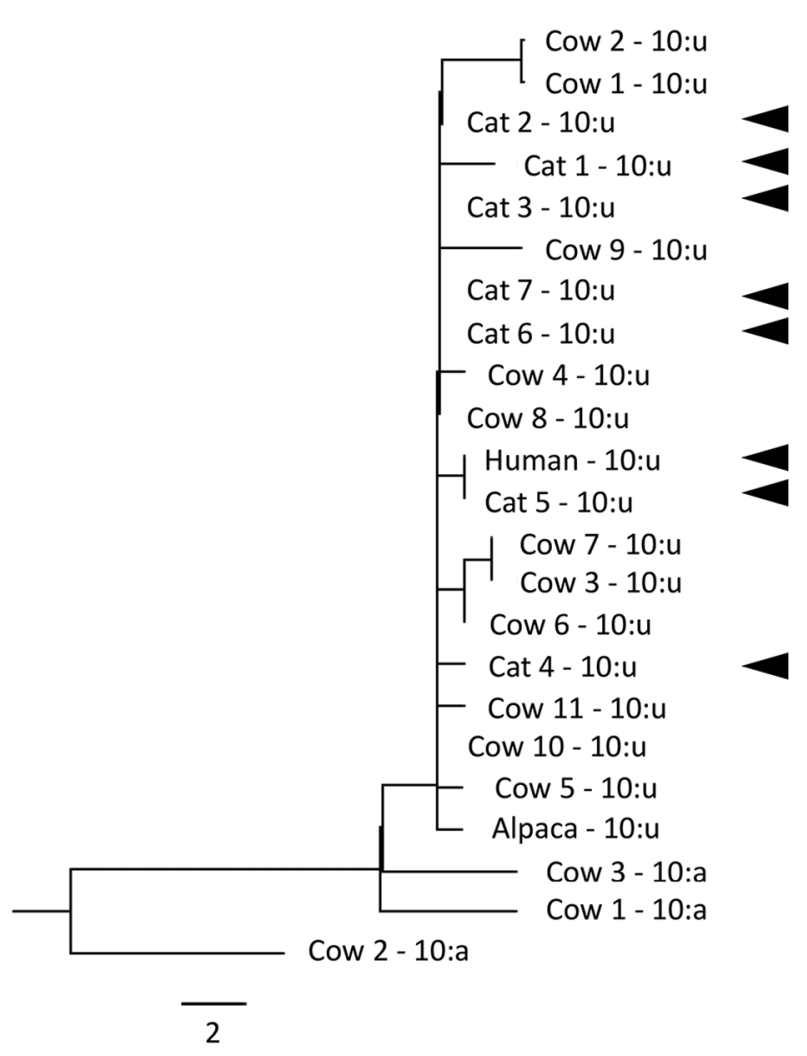

Figure. Whole-genome sequencing phylogenetic relationship of genotype 10:u isolates of Mycobacterium bovis from 1 human, 7 cats, 11 cattle, and 1 alpaca, and 10:a isolates from 3 cattle (maximum-likelihood tree of all single-nucleotide polymorphisms [SNPs]). Cat and human isolates are indicated by solid arrowheads. Heat-killed cultures were sequenced by using a MiSeq Sequencer (Illumina, https://www.illumina.com), and reads were mapped by using reference strain AF2122. The average coverage ranged from 23.9-fold to 88.8-fold. The human and their household cat contact (cat 5) isolates were indistinguishable in their genome sequences. Scale bar indicates SNPS.

by aerosols from cats with respiratory signs or aerosolgenerating procedures.

Public Health England now advises that all close contacts of household companion animals with confirmed $M$. bovis infections should be assessed by a public health professional and receive guidance on how to best minimize zoonotic transmission $(12,13)$. In addition, as part of an enhanced surveillance system in England and Wales, newly diagnosed human case-patients with M. bovis infection are now also asked explicitly about contact with pets with suspected or confirmed $M$. bovis disease (14).

In summary, M. bovis disease in companion animals, particularly cats with severe systemic features including exudative lesions, can no longer be regarded as posing a negligible public health risk. Guidance should be provided to minimize the risk for transmission to human contacts. 


\section{Acknowledgments}

We thank Kate McPhedran for assistance at the time of the incidents and Karen Gover and Monika Klita for assistance in testing and analyzing human and animal isolates.

\section{About the Author}

Ms. O'Connor is an epidemiologist in the National Infection Service at Public Health England, London, UK. Her primary research interests are epidemic intelligence, risk assessment, and emerging zoonotic infections.

\section{References}

1. Public Health England. Tuberculosis caused by Mycobacterium bovis (M. bovis): notification data; 2018 [cited 2018 Nov 13]. https://www.gov.uk/government/publications/mycobacteriumbovis-mbovis-tuberculosis-annual-data

2. Davidson JA, Loutet MG, O'Connor C, Kearns C, Smith RM, Lalor MK, et al. Epidemiology of Mycobacterium bovis disease in Humans in England, Wales, and Northern Ireland, 2002-2014. Emerg Infect Dis. 2017;23:377-86. https://doi.org/10.3201/ eid2303.161408

3. Department for Environment, Food, and Rural Affairs. Tuberculosis (TB) in cattle in Great Britain (update 17 October 2018); 2018 [cited 2018 Nov 13]. https://www.gov.uk/government/ statistical-data-sets/tuberculosis-tb-in-cattle-in-great-britain

4. Department for Environment, Food, and Rural Affairs. Incidents of $M$. bovis infection in non-bovine domestic animals and wild deer in GB confirmed by laboratory culture; 2015 [cited 2018 Dec 3]. www.gov.uk/government/uploads/system/uploads/attachment_data/ file/232602/bovinetb-otherspecies-27aug13.xls

5. Gunn-Moore DA, McFarland SE, Brewer JI, Crawshaw TR, Clifton-Hadley RS, Kovalik M, et al. Mycobacterial disease in cats in Great Britain: I. Culture results, geographical distribution and clinical presentation of 339 cases. J Feline Med Surg. 2011;13:934 44. https://doi.org/10.1016/j.jfms.2011.07.012
6. Ramdas KE, Lyashchenko KP, Greenwald R, Robbe-Austerman S, McManis C, Waters WR. Mycobacterium bovis infection in humans and cats in same household, Texas, USA, 2012. Emerg Infect Dis. 2015;21:480-3. https://doi.org/10.3201/eid2103.140715

7. Lewis-Jonsson J. Transmission of tuberculosis from cats to human beings. Acta Tuberculosea et Pneumologica Scandinavica. 1946;20:102.

8. Roberts T, O'Connor C, Nuñez-Garcia J, de la Rua-Domenech R, Smith NH. Unusual cluster of Mycobacterium bovis infection in cats. Vet Rec. 2014;174:326. https://doi.org/10.1136/vr.102457

9. Public Health England. Tuberculosis screening; 2018 [cited 2019 Jul 30]. https://www.gov.uk/guidance/tuberculosis-screening

10. Talwar A, McGown A, Langham T, Abid M, Pilling J; Department of Respiratory Medicine, Royal Berkshire Hospital. A very strange tail. Thorax. 2014;69:1159-60. https://doi.org/10.1136/thoraxjnl-2014-206033

11. Human Animal Infections and Risk Surveillance group (HAIRS). Qualitative assessment of the risk that cats infected with Mycobacterium bovis present to human health; 2014 [cited 2018 Dec 3]. https://www.gov.uk/government/publications/hairs-riskassessment-mycobacterium-bovis-in-cats

12. Public Health England. Bovine tuberculosis: guidance on management of the public health consequences of tuberculosis in cattle and other animals (England); 2014 [cited 2018 Dec 3]. https:/www.gov.uk/government/publications/bovine-tuberculosistb-public-health-management

13. UK Government. Bovine tuberculosis in domestic pets; 2014 [cited $2019 \mathrm{Jul} \mathrm{30]}$. https://assets.publishing.service.gov.uk/government/uploads/system/uploads/attachment_data/file/596240/AG-TBYP-01e.pdf

14. Public Health England. Mycobacterium bovis (M. bovis): enhanced surveillance questionnaire; 2016 [cited 2018 Dec 3]. https://www.gov.uk/government/publications/mycobacteriumbovis-m-bovis-enhanced-surveillance-questionnaire

Address for correspondence: Catherine M. O'Connor, TARGET, Emerging Infections and Zoonoses Team, National Infection Service, Public Health England, 61 Colindale Ave, London NW9 5EQ, UK; email: catherine.oconnor@phe.gov.uk

\section{EID Podcast:}

\section{Veterinarian Gets Flu Virus from Cats}

Avian influenza viruses occasionally cross the species barrier, infecting humans and other mammals after exposure to infected birds and contaminated environments. Unique among the avian influenza $A$ subtypes, both low pathogencity and highly pathogenic $\mathrm{H} 7$ viruses have demonstrated the ability to infect and cause disease in humans.

In this podcast, Dr. Todd Davis, a CDC research biologist, discusses transmission of avian H7N2 from a cat to a human. 